\title{
Investigating the phenotypic and genetic associations between personality traits and suicidal behavior across major mental health diagnoses
}

\author{
Janos L. Kalman ${ }^{1,2,3}$ (D) Tomoya Yoshida ${ }^{4}$ Till F. M. Andlauer ${ }^{5,6} \cdot$ Eva C. Schulte $^{1,2} \cdot$ Kristina Adorjan $^{1,2}$. \\ Martin Alda ${ }^{7}$ Raffaela Ardau ${ }^{8}$. Jean-Michel Aubry ${ }^{9,10}$. Katharina Brosch ${ }^{11,12}$. Monika Budde ${ }^{1}$. \\ Caterina Chillotti ${ }^{8}$ - Piotr M. Czerski ${ }^{13} \cdot$ Raymond J. DePaulo $^{14}$. Andreas Forstner ${ }^{15,16,17}$. Fernando S. Goes ${ }^{14}$. \\ Maria Grigoroiu-Serbanescu ${ }^{18}$. Paul Grof ${ }^{19,20}$. Dominik Grotegerd ${ }^{21}$. Tim Hahn ${ }^{21}$. Maria Heilbronner ${ }^{1}$. \\ Roland Hasler ${ }^{9}$. Urs Heilbronner ${ }^{1}$. Stefanie Heilmann-Heimbach ${ }^{16}$. Pawel Kapelski ${ }^{13}$. Tadafumi Kato $^{22}$. \\ Mojtaba Oraki Kohshour ${ }^{1,23}$. Susanne Meinert ${ }^{21,24} \cdot$ Tina Meller $^{11,12} \cdot$ Igor Nenadić $^{11,12} \cdot$ Markus M. Nöthen $^{16}$. \\ Tomas Novak ${ }^{25,26}$. Nils Opel ${ }^{21}$. Joanna Pawlak ${ }^{13}$. Julia-Katharina Pfarr ${ }^{11,12}$. James B. Potash ${ }^{14}$. \\ Daniela Reich-Erkelenz ${ }^{1}$. Jonathan Repple ${ }^{21} \cdot$ Hélène Richard-Lepouriel $I^{9} \cdot$ Marcella Rietschel $^{27}$. \\ Kai G. Ringwald ${ }^{11,12}$. Guy Rouleau ${ }^{28}$. Sabrina Schaupp ${ }^{1} \cdot$ Fanny Senner $^{1,2} \cdot$ Giovanni Severino $^{29}$. \\ Alessio Squassina ${ }^{29}$. Frederike Stein ${ }^{11,12} \cdot$ Pavla Stopkova $^{25,26} \cdot$ Fabian Streit $^{27} \cdot$ Katharina Thiel $^{21}$. \\ Florian Thomas-Odenthal ${ }^{11}$. Gustavo Turecki ${ }^{30}$. Joanna Twarowska-Hauser ${ }^{13}$ - Alexandra Winter ${ }^{21} \cdot$ Peter P. Zandi $^{14}$. \\ John R. Kelsoe ${ }^{31}$. Consortium on Lithium Genetics (ConLiGen), PsyCourse · Peter Falkai ${ }^{2}$. Udo Dannlowski ${ }^{21}$. \\ Tilo Kircher ${ }^{11,12} \cdot$ Thomas G. Schulze ${ }^{1,14,32} \cdot$ Sergi Papiol ${ }^{1,2,33}$
}

Received: 12 October 2021 / Accepted: 24 November 2021 / Published online: 10 February 2022

(c) The Author(s) 2022

\begin{abstract}
Personality traits influence risk for suicidal behavior. We examined phenotype- and genotype-level associations between the Big Five personality traits and suicidal ideation and attempt in major depressive, bipolar and schizoaffective disorder, and schizophrenia patients $(N=3012)$ using fixed- and random-effects inverse variance-weighted meta-analyses. Suicidal ideations were more likely to be reported by patients with higher neuroticism and lower extraversion phenotypic scores, but showed no significant association with polygenic load for these personality traits. Our findings provide new insights into the association between personality and suicidal behavior across mental illnesses and suggest that the genetic component of personality traits is unlikely to have strong causal effects on suicidal behavior.
\end{abstract}

Keywords Suicidal behavior $\cdot$ Personality $\cdot$ Polygenic score $\cdot$ Bipolar disorder $\cdot$ Major depression $\cdot$ Schizophrenia

\section{Introduction}

Suicide is a leading cause of mortality [1], and most individuals with suicidal behavior, which includes suicidal ideation (SI), suicide attempt (SA), and completed suicide, have

Thomas G. Schulze and Sergi Papiol are joint senior authors.

The members of the International Consortium on Lithium Genetics and PsyCourse Group are mentioned "Acknowledgments section".

Janos L. Kalman

janos.kalman@med.uni-muenchen.de

Extended author information available on the last page of the article a diagnosed mental health disorder [2].

Suicidal behavior has a complex, heterogenous etiology. Its risk factors include genetics, personality characteristics, and adverse life events [3]. Twin and family studies showed that suicidal behavior is heritable and that $30-55 \%$ of its phenotypic variance is explained by genetic risk factors that only partially overlap with those for mental disease [4-7]. Although individual predictors explain only a fraction of the phenotypic variability, studying them is useful to enhance our understanding of disease pathophysiology and inform the development of diagnostic and preventive measures [8].

Personality characteristics, like the Big Five personality traits (neuroticism, agreeableness, conscientiousness, extraversion, openness), or TEMPS-A temperaments are 
relatively stable throughout life $[9,10]$. They influence the perception of and exposure and response to life events and thus mediate susceptibility and/or resilience to environmental risk factors. For example, individuals with higher neuroticism show high emotional arousal, experience more negative emotions and are more sensitive to negative emotional stimuli and potential loss $[10,11]$. In contrast, high extraversion is associated with higher levels of energy and sociability and more positive affect [12]. Hence, multiple studies have provided compelling evidence that high neuroticism and low extraversion are important risk factors for suicidal behavior [13-15]. A substantial amount of the phenotypic variance of these personality traits is explained by common genetic variants: single-nucleotide polymorphism (SNP)-based heritability estimates range from 6 to $15 \%$ for neuroticism and $5 \%$ to $18 \%$ for extraversion $[11,12,16,17]$.

The polygenic makeup of personality traits is one of the few quantifiable biological factors that likely influences suicidal behavior $[11,12,18]$, so it is relevant to investigate how much phenotypic variance in suicidal behavior is explained by the polygenic load for personality traits [19]. Furthermore, it would be important to understand whether personality traits have a disease-specific or cross-diagnostic influence on suicidal behavior risk in mental illness. Therefore, we investigated $a$ ) the association between personality traits and SI and SA across the affective-psychotic diagnosis spectrum, $b$ ) whether associations differ between diagnostic groups, and $c$ ) what percentage of phenotypic variation in suicidal behavior is attributable to polygenic scores (PGS) for personality traits.

\section{Patients and methods}

\section{Sample description}

Participants with DSM-IV diagnosis of major depressive disorder (MDD), bipolar disorder (BD), schizoaffective disorder (SCZA), or schizophrenia (SCZ) and available information on lifetime SI or SA (presence/absence) and genetic data were selected from nine independent datasets of European-ancestry cases $(N=3012)$. Sample details, including the definitions of suicidal behavior, are described in the Supplementary Information, including Supplementary Table S4.

The Big Five model is the most widely accepted personality theory, and it is extensively used in research. Two samples (PsyCourse and FOR2107) included individual-level information on the Big Five personality traits, assessed with either the short version of the Big Five Inventory (PsyCourse) or the NEO Five Factor Inventory (FOR2107; Supplementary Table S6) [20, 21].

\section{Genetic analyses}

The cohorts were genotyped by different microarray types in accordance with local protocols. Quality control and population substructure analyses were performed with PLINK v1.9 and either $R$ (for the PsyCourse and FOR2107 cohorts) or the RICOPILI pipeline (for the other seven cohorts), as described previously [22-24] (Supplementary Methods and Supplementary Tables S1-S2). Imputation was performed with SHAPEIT and IMPUTE2 with the 1000 Genomes Phase 3 (for the FOR2107 and PsyCourse cohorts) or the Haplotype Reference Consortium v1.0 (for the other cohorts) reference panels. For our analyses, we selected variants present in the PRS-CS 1000 Genomes Phase 3 EUR reference dataset.

PRS-CS was used to calculate PGS for personality traits with significant effects at the phenotype level by using summary statistics from genome-wide association studies as training datasets (Supplementary Table S3) [11, 12, 25].

\section{Statistical analyses}

In the primary phenotype-level analyses, we analyzed the association of personality traits with SI and SA within the PsyCourse and FOR2017 samples by logistic regression, with sex, age, and BD subtype as covariates. Results of these analyses were meta-analyzed using fixed- and randomeffects inverse variance-weighted meta-analyses. Potential subgroup effects specific for DSM categories were investigated with diagnosis-specific meta-analyses. To investigate associations of extraversion and neuroticism PGS with SI and SA, we used the same analysis models as for the phenotype-level analyses. Genotyping batch (for the Romanial and euoR samples) and the first eight multidimensional scaling ancestry components were used as additional covariates. The statistical power of our sample was estimated with $\mathrm{G}^{*}$ Power 3.1 and the avengeme $\mathrm{R}$ package [26, 27]. The significance threshold was corrected for 14 tests by Bonferroni's method $(\alpha=0.05 /[5$ personality traits $\times 2$ phenotypes and $2 \mathrm{PGS} \times 2$ phenotypes] $=3.57 \times 10^{-3}$ ).

\section{Results}

The frequency of SI and SA in our study was $61.16 \%$ and $31.28 \%$, respectively.

\section{Personality and suicidal behavior}

Our study had $80 \%$ power $(\alpha=0.05)$ to detect effects of personality traits with odds ratio $(\mathrm{OR}) \geq 1.15$ on SI and $\mathrm{OR} \geq 1.23$ on SA. 
In the fixed-effects meta-analysis of the FOR2107 and PsyCourse samples, neuroticism was significantly associated with an increased likelihood of SI $(\mathrm{OR}=1.37,95 \%$ CI [1.23-1.54], $p=2.11 \times 10^{-8}$, Cochran's $Q p=0.02$, $I^{2}=57.9 \%$ ), and extraversion, with a decreased likelihood $\left(\mathrm{OR}=0.78,95 \%\right.$ CI $[0.70-0.87], p=1.01 \times 10^{-5}$, Cochran's $Q p=0.06, I^{2}=48.3 \%$ ) (Table 1, Fig. 1). The randomeffects meta-analysis results were not substantially different (Table 1). In the secondary, diagnosis-specific meta-analyses, the effect direction was consistent across diagnostic groups, although a high level of heterogeneity (Cochran's $Q p<0.05$ ) was observed in all diagnoses except MDD and partially SCZA (Supplementary Table S7). After correction for multiple testing, none of the other personality traits showed significant associations (Table 1).

None of the personality traits was significantly associated with SA, although the direction of the effects was the same as with SI (Table 1).

\section{PGS for personality traits and suicidal behavior}

No significant association was found between neuroticism and extraversion PGS and SI or SA (Table 1). Post hoc power analyses indicated that none of the PGS analyses in our sample had $80 \%$ power to identify PGS effects with $p<0.05$.

\section{Discussion}

To our knowledge, this study is the first attempt to dissect the phenotypic and genetic relationship between personality traits and suicidal behavior across the affective-psychotic diagnostic spectrum. We found significant phenotype-level associations of both neuroticism and extraversion-two personality traits known to influence affect processing —with SI across diagnostic groups but no evidence that these associations were driven by the polygenic load for these traits.

An association of neuroticism with increased suicidal behavior risk has already been described in population-based cohorts [14, 28, 29] and studies on individuals with personality [30] or affective disorders [31-33]; our secondary analyses confirmed these findings in patients with MDD (the largest diagnostic group in our study) and showed similar effects for BD and SCZ, suggesting that neuroticism may represent a transdiagnostic risk factor for SI [34].

Studies reported a protective effect of extraversion in the general population $[15,35]$ and patients with affective disorder $[33,35]$. The diagnosis-specific results in our study support such a protective effect in MDD and suggest similar effects in BD.
Although our SA sample was sufficiently powered to detect effect sizes comparable to those observed for SI, we found no significant associations of neuroticism and extraversion with $\mathrm{SA}$, which is a more severe phenotype than SI. This finding suggests a lesser involvement, or a lack thereof, of personality traits in SA in comparison with SI.

The present study constitutes, to our knowledge, the first attempt to ascertain the role of a polygenic load associated with personality traits on suicidal behavior in a sample exclusively composed of patients with psychiatric diagnoses. Despite the phenotype-level associations, neuroticism and extraversion PGS were not significantly associated with suicide-related phenotypes, which contrasts with a study that detected an association between neuroticism PGS and SA or self-harm in a populationbased cohort of 4959 individuals [36]. However, according to our post-hoc analysis, our study lacked statistical power to replicate these findings. Furthermore, neuroticism and extraversion PGS explained only a small proportion of the phenotypic variance of the respective personality traits in our study $\left(R_{\text {neuroticism }}^{2}=0.011, R_{\text {extraversion }}^{2}=0.0059\right)$.

\section{Limitations}

The heterogeneous definitions of personality, SI, and SA in the samples, as also implied by the heterogeneity estimates $\left(I^{2}\right)$ of our analyses, are an important limitation. SI and SA are broad concepts with no universally accepted definitions [37]. Accordingly, their prevalence might be impacted by cohort-specific differences, as also observed in our study (Supplementary Tables S4, S6). A further potential source of heterogeneity was the use of different questionnaires to assess personality traits in the various cohorts. Notably, these issues represent a general problem in psychiatric research [38]. To assess the effect of heterogeneity on our results, we performed random- and fixed-effects meta-analyses. Another limitation is that we did not account for possible confounders by assessing environmental precipitating factors. Last, our sample had limited statistical power, which reduced the likelihood of detecting true-positive signals.

\section{Conclusion}

Our findings reinforce the notion that personality traits contribute to the expression of SI independently of diagnosis, and they provide preliminary evidence that personality trait PGS are unlikely to have strong causal effects on suicidal behavior. These findings need validation in larger clinical datasets. 


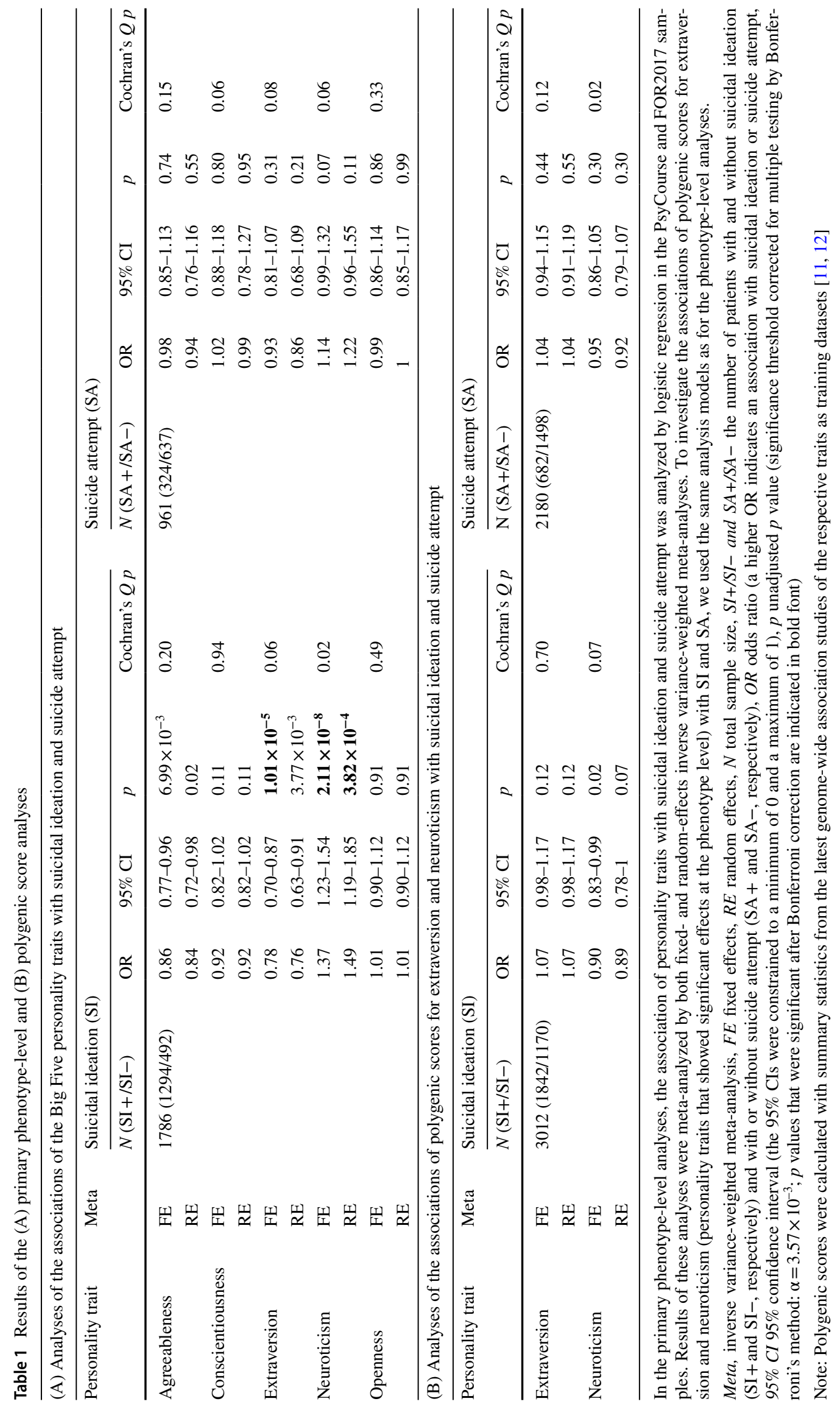




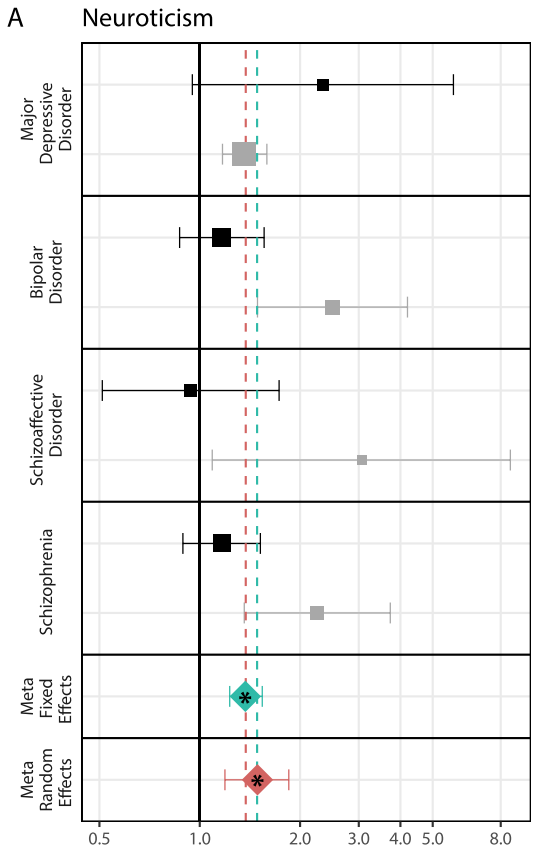

OR for suicidal ideation $+95 \% \mathrm{Cl}(\log 10$ scale)
B

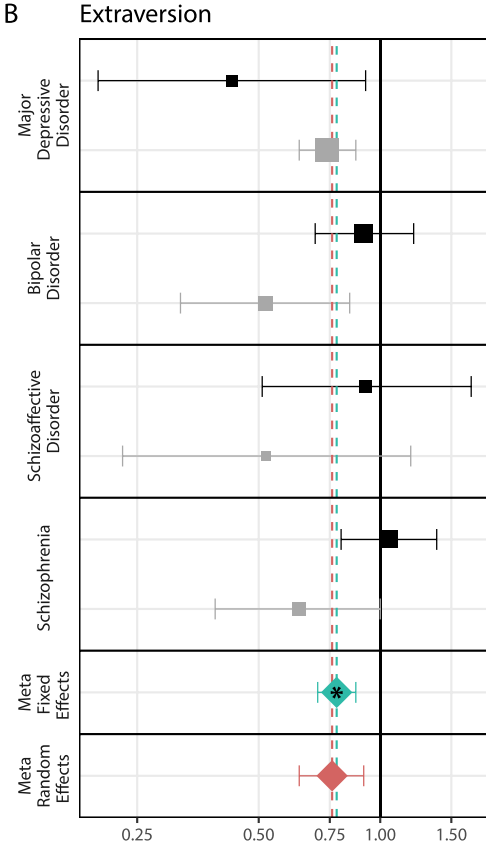

OR for suicidal ideation $+95 \% \mathrm{Cl}(\log 10$ scale $)$

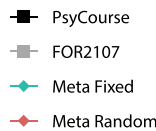

- Meta Random
Fig. 1 Results of secondary phenotype-level analyses. For extraversion and neuroticism, which were significantly associated with suicidal ideation in the primary analyses, we conducted secondary analyses to investigate potential differences across the diagnostic spectrum. Results of the individual regression models can be found in Supplementary Table S7. Inverse variance-weighted metaanalysis $p$ values that were significant after Bonferroni correction $\left(\alpha<3.57 \times 10^{-3}\right)$ are indicated with an asterisk. Meta fixed, inverse variance-weighted fixed-effects meta-analysis; meta random, inverse variance-weighted random-effects meta-analysis; 95\% CI, 95\% confidence intervals (the $95 \%$ CIs were constrained to a minimum of 0 and a maximum of 1); OR, odds ratio (a higher OR indicates an association with suicidal ideation

Canada; L. Trevor Young, Department of Psychiatry, University of British Columbia, Vancouver, Canada; Carlos A. López Jaramillo, Department of Psychiatry, University of Antioquia, Medellín, Medellín, Colombia; Tomás Novák, Prague Psychiatric Center and 3rd Faculty of Medicine, Charles University, Prague, Czech Republic; Pavla Stopkova, Prague Psychiatric Center and 3rd Faculty of Medicine, Charles University, Prague, Czech Republic; Frank Bellivier, INSERM UMR-S 1144 - Pôle de Psychiatrie, AP-HP, Groupe Hospitalier Lariboisière-F. Widal, Paris, France; Clara Brichant-Petitjean, INSERM UMR-S 1144 - Pôle de Psychiatrie, AP-HP, Groupe Hospitalier Lariboisière-F. Widal, Paris, France; Bruno Etain, Inserm U955, Psychiatrie Génétique, Créteil, France; Bruno Etain, Université Paris Est, Faculté de Médecine, Créteil, France ; Bruno Etain, Fondation FondaMental, Créteil, France ; Bruno Etain, Assistance Publique-Hôpitaux de Paris, Hôpital Albert Chenevier - Henri Mondor, Pôle de Psychiatrie, Créteil, France; Sébastien Gard, Service de psychiatrie, Hôpital Charles Perrens, Bordeaux, France ; Stéphane Jamain, Inserm U955, Psychiatrie Génétique, Créteil, France; Stéphane Jamain, Université Paris Est, Faculté de Médecine, Créteil, France; Stéphane Jamain, Fondation FondaMental, Créteil, France ; Jean-Pierre Kahn, Service de Psychiatrie et Psychologie Clinique, Centre Hospitalier Universitaire de Nancy, Nancy, France ; Jean-Pierre Kahn, Université de Lorraine, Nancy, France ; Marion Leboyer, Inserm U955, Psychiatrie Génétique, Créteil, France; Marion Leboyer, Université Paris Est, Faculté de Médecine, Créteil, France ; Marion Leboyer, Fondation FondaMental, Créteil, France; Marion Leboyer, Assistance Publique-Hôpitaux de Paris, Hôpital Albert Chenevier - Henri Mondor, Pôle de Psychiatrie, Créteil, France; Mazda Adli, Department of Psychiatry and Psychotherapy, Charité - Universitätsmedizin Berlin, Campus Charité Mitte \& Fliedner Klinik Berlin, Germany; Mazda Adli, Fliedner Klinik Berlin, Berlin, Dalhousie University, Halifax, Nova Scotia, Canada; Gustavo Turecki, Douglas Mental Health University Institute, McGill University, Montreal, 
Germany; Michael Bauer, Department of Psychiatry and Psychotherapy, University Hospital Carl Gustav Carus, Technische Universität Dresden, Dresden, Germany; Sven Cichon, Institute of Human Genetics, Department of Genomics, Life and Brain Center, University of Bonn, Bonn, Germany; Sven Cichon, Institute of Neuroscience and Medicine (INM-1), Genomic Imaging, Research Center Juelich, Juelich, Germany; Franziska Degenhardt, Institute of Human Genetics, Department of Genomics, Life and Brain Center, University of Bonn, Bonn, Germany; Peter Falkai, Department of Psychiatry and Psychotherapy, Ludwig-MaximiliansUniversity Munich, Munich, Germany; Oliver Gruber, Department of Psychiatry and Psychotherapy, Georg-August University Göttingen, Göttingen, Germany; Urs Heilbronner, Department of Psychiatry and Psychotherapy, Georg-August University Göttingen, Göttingen, Germany; Per Hoffmann, Institute of Human Genetics, Department of Genomics, Life and Brain Center, University of Bonn, Bonn, Germany; Per Hoffmann, Institute of Neuroscience and Medicine (INM-1), Genomic Imaging, Research Center Juelich, Juelich, Germany; Sarah Kittel-Schneider, Department of Psychiatry, Psychosomatics, and Psychotherapy, University of Würzburg, Würzburg, Germany; Markus Nöthen, Institute of Human Genetics, Department of Genomics, Life and Brain Center, University of Bonn, Bonn, Germany; Andrea Pfennig, Department of Psychiatry and Psychotherapy, University Hospital Carl Gustav Carus, Technische Universität Dresden, Dresden, Germany; Daniela Reich-Erkelenz, Department of Psychiatry and Psychotherapy, Ludwig-Maximilians-University Munich, Munich, Germany; Andreas Reif, Department of Psychiatry, Psychosomatics, and Psychotherapy, University of Würzburg, Würzburg, Germany; Marcella Rietschel, Department of Genetic Epidemiology in Psychiatry, Central Institute of Mental Health, Mannheim, Germany; Thomas G. Schulze, Department of Psychiatry and Psychotherapy, Georg-August University Göttingen, Göttingen, Germany; Florian Seemüller, Department of Psychiatry and Psychotherapy, Ludwig-Maximilians-University Munich, Munich, Germany; Thomas Stamm, Department of Psychiatry and Psychotherapy, Charité - Universitätsmedizin Berlin, Campus Charité Mitte, Berlin, Germany; Raffaella Ardau, Unit of Clinical Pharmacology, Hospital University Agency, University of Cagliari, Cagliari, Italy; Caterina Chillotti, Unit of Clinical Pharmacology, Hospital University Agency, University of Cagliari, Cagliari, Italy; Maria Del Zompo, Department of Biomedical Sciences, University of Cagliari, Cagliari, Italy; Maria Del Zompo, Unit of Clinical Pharmacology, Hospital University Agency, University of Cagliari, Cagliari, Italy; Mario Maj, Department of Psychiatry, University of Naples, SUN, Naples, Italy; Mirko Manchia, Department of Biomedical Sciences, University of Cagliari, Cagliari, Italy; Palmiero Monteleone, Department of Psychiatry, University of Naples, SUN, Naples, Italy; Giovanni Severino, Department of Biomedical Sciences, University of Cagliari, Cagliari, Italy; Alessio Squassina, Department of Biomedical Sciences, University of Cagliari, Cagliari, Italy; Alfonso Tortorella, Department of Psychiatry, University of Naples, SUN, Naples, Italy; Kazufumi Akiyama, Department of Biological Psychiatry and Neuroscience, Dokkyo Medical University School of Medicine, Mibu, Japan; Kazufumi Akiyama, The Japanese Collaborative Group on the Genetics of Lithium Response in Bipolar Disorder, Japan; Ryota Hashimoto, Molecular Research Center for Children's Mental Development, United Graduate School of Child Development, Osaka University, Osaka University, Osaka, Japan; Ryota Hashimoto, The Japanese Collaborative Group on the Genetics of Lithium Response in Bipolar Disorder, Japan; Tadafumi Kato, The Japanese Collaborative Group on the Genetics of Lithium Response in Bipolar Disorder, Japan; Tadafumi Kato, Laboratory for Molecular Dynamics of Mental Disorders, RIKEN Brain Science Institute, Saitama, Japan; Ichiro Kusumi, The Japanese Collaborative Group on the Genetics of Lithium Response in Bipolar Disorder, Japan; Ichiro Kusumi, Department of Psychiatry, Hokkaido University Graduate School of Medicine, Sapporo, Japan; Takuya Masui, The Japanese Collaborative Group on the Genetics of Lithium Response in Bipolar Disorder, Japan; Takuya Masui, Department of Psychiatry, Hokkaido University Graduate School of Medicine, Sapporo, Japan; Norio Ozaki, The
Japanese Collaborative Group on the Genetics of Lithium Response in Bipolar Disorder, Japan; Norio Ozaki, Department of Psychiatry, Nagoya University Graduate School of Medicine, Nagoya, Japan; Piotr Czerski, Psychiatric Genetic Unit, Poznan University of Medical Sciences, Poznan, Poland; Joanna Hauser, Psychiatric Genetic Unit, Poznan University of Medical Sciences, Poznan, Poland; Sebastian Kliwicki, Department of Adult Psychiatry, Poznan University of Medical Sciences, Poznan, Poland; Janusz K. Rybakowski, Department of Adult Psychiatry, Poznan University of Medical Sciences, Poznan, Poland; Maria Grigoroiu-Serbanescu, Biometric Psychiatric Genetics Research Unit, Alexandru Obregia Psychiatric Hospital, Bucharest, Romania; Bárbara Arias, Department of Biologia Animal, Unitat d'Antropologia, Facultat de Biologia, Universitat de Barcelona, IBUB, CIBERSAM, Instituto de Salud Carlos III, BarceIona, Catalonia, Spain; Antonio Benabarre, Bipolar Disorders Program, Institute of Neuroscience, Hospital Clinic, University of Barcelona, IDIBAPS, CIBERSAM, Barcelona, Catalonia, Spain; Francesc Colom, Bipolar Disorders Program, Institute of Neuroscience, Hospital Clinic, University of Barcelona, IDIBAPS, CIBERSAM, Barcelona, Catalonia, Spain; Esther Jiménez, Bipolar Disorders Program, Institute of Neuroscience, Hospital Clinic, University of Barcelona, IDIBAPS, CIBERSAM, Barcelona, Catalonia, Spain; Marina Mitjans, Department of Biologia Animal, Unitat d'Antropologia, Facultat de Biologia, Universitat de Barcelona, IBUB, CIBERSAM, Instituto de Salud Carlos III, Barcelona, Catalonia, Spain; Eduard Vieta, Bipolar Disorders Program, Institute of Neuroscience, Hospital Clinic, University of Barcelona, IDIBAPS, CIBERSAM, Barcelona, Catalonia, Spain; Lena Backlund, Department of Molecular Medicine and Surgery, Karolinska Institutet and Center for Molecular Medicine, KarolinskaUniversity Hospital, Stockholm, Sweden; Lena Backlund, Department of ClinicalNeuroscience, Centre for Psychiatric Research and Education, Karolinska Institutet, The Clinic for Affective Disorders, Karolinska University Hospital, Stockholm, Sweden; Louise Frisén, Department of Molecular Medicine and Surgery, Karolinska Institutet and Center for Molecular Medicine, Karolinska University Hospital, Stockholm, Sweden Louise Frisén, Department of Clinical Neuroscience, Centre for Psychiatric Research and Education, Karolinska Institutet, The Clinic for Affective Disorders, Karolinska University Hospital, Stockholm, Sweden; Catharina Lavebratt, Department of Molecular Medicine and Surgery, Karolinska Institutet and Center for Molecular Medicine, Karolinska University Hospital, Stockholm, Sweden Lina Martinsson, Department of Molecular Medicine and Surgery, Karolinska Institutet and Center for Molecular Medicine, Karolinska University Hospital, Stockholm, Sweden; Lina Martinsson, Department of Clinical Neuroscience, Centre for Psychiatric Research and Education, Karolinska Institutet, The Clinic for Affective Disorders, Karolinska University Hospital, Stockholm, Sweden; Urban Ösby, Department of Molecular Medicine and Surgery, Karolinska Institutet and Center for Molecular Medicine, Karolinska University Hospital, Stockholm, Sweden; Martin Schalling, Department of Molecular Medicine and Surgery, Karolinska Institutet and Center for Molecular Medicine, Karolinska University Hospital, Stockholm, Sweden; Jean-Michel Aubry, Départment de Psychiatrie, HUG - Hôpitaux Universitaires de Genève, Geneva, Switzerland; Sven Cichon, Division of Medical Genetics, Department of Biomedicine, University of Basel, Basel, Switzerland; Alexandre Dayer, Départment de Psychiatrie, HUG - Hôpitaux Universitaires de Genève, Geneva, Switzerland; Alexandre Dayer, Department of Basic Neurosciences, University of Geneva Medical School, Geneva, Switzerland ; Per Hoffmann, Division of Medical Genetics, Department of Biomedicine, University of Basel, Basel, Switzerland; Audrey Nallet, Department of Mental Health and Psychiatry, Hôpitaux Universitaires de Genéve, Geneva, Switzerland ; Hsi-Chung Chen, Department of Psychiatry \& Center of Sleep Disorders, National Taiwan University Hospital, Taipei, Taiwan; Po-Hsiu Kuo, Institute of Epidemiology and Preventive Medicine, National Taiwan University, Taipei, Taiwan; David Cousins, Campus for Ageing and Vitality, Newcastle University, Newcastle, United Kingdom; Nirmala Akula, Human Genetics Branch, National Institute of Mental Health and Human Services, Bethesda, MD, United States; Joanna M. Biernacka, 
Department of Psychiatry and Psychology, Mayo Clinic, Rochester, MN, United States; Joanna M. Biernacka, Department of Health Sciences Research, Mayo Clinic, Rochester, MN, United States; Elise T. Bui, Human Genetics Branch, National Institute of Mental Health and Human Services, Bethesda, MD, United States; J. Ray DePaulo, Department of Psychiatry and Behavioral Sciences, Johns Hopkins University, Baltimore, MD, United States; Sevilla D. Detera-Wadleigh, Human Genetics Branch, National Institute of Mental Health and Human Services, Bethesda, MD, United States; Mark A. Frye, Department of Psychiatry and Psychology, Mayo Clinic, Rochester, MN, United States; Fernando S. Goes, Department of Psychiatry and Behavioral Sciences, Johns Hopkins University, Baltimore, MD, United States; Rebecca Hoban, Department of Psychiatry, University of California San Diego, United States; Rebecca Hoban, Department of Psychiatry, VA San Diego Healthcare System, United States; Liping Hou, Human Genetics Branch, National Institute of Mental Health and Human Services, Bethesda, MD, United States; Layla Kassem, Human Genetics Branch, National Institute of Mental Health and Human Services, Bethesda, MD, United States; John R. Kelsoe, Department of Psychiatry, University of California San Diego, San Diego, CA, United States; John R. Kelsoe, Department of Psychiatry, VA San Diego Healthcare System, San Diego, CA, United States; Gonzalo Laje, Human Genetics Branch, National Institute of Mental Health and Human Services, Bethesda, MD, United States; Gonzalo Laje, Washington Behavioral Medicine Associates, LLC, Chevy Chase, MD, United States; Gonzalo Laje, Maryland Institute for Neuroscience \& Development (MIND), Chevy Chase, MD, United States; Susan G. Leckband, Department of Psychiatry, University of California San Diego, San Diego, CA, United States; Susan G. Leckband, Department of Pharmacy, VA San Diego Healthcare System, San Diego, CA, United States; Susan G. Leckband, Skaggs School of Pharmacy and Pharmaceutical Sciences, University of California, San Diego, CA, United States; Michael J. McCarthy, Department of Psychiatry, VA San Diego Healthcare System, San Diego, CA, United States; Francis J. McMahon, Human Genetics Branch, National Institute of Mental Health and Human Services, Bethesda, MD, United States; Francis Mondimore, Department of Psychiatry and Behavioral Sciences, Johns Hopkins University, Baltimore, MD, United States; Roy H. Perlis, Department of Psychiatry, Massachusetts General Hospital and Harvard Medical School, Boston, MA, United States; James B. Potash, Department of Psychiatry, University of lowa, lowa City, IA, United States; Thomas G. Schulze, Department of Psychiatry and Behavioral Sciences, Johns Hopkins University, Baltimore, MD, United States; Thomas G. Schulze, Human Genetics Branch, National Institute of Mental Health and Human Services, Bethesda, MD, United States; Barbara Schweizer, Department of Psychiatry and Behavioral Sciences, Johns Hopkins University, Baltimore, MD, United States; Lisa R. Seymour, Department of Psychiatry, Mayo Clinic, Rochester, MN, United States; Jordan W. Smoller, Department of Psychiatry, Massachusetts General Hospital and Harvard Medical School, Boston, MA, United States; Jo Steele, Human Genetics Branch, National Institute of Mental Health and Human Services, Bethesda, MD, United States; Sarah Tighe, Department of Psychiatry, University of lowa, lowa City, IA, United States; Peter P. Zandi, Department of Mental Health, Johns Hopkins Bloomberg School of Public Health, Baltimore, MD, United States. Authors of the PsyCourse Group: Adorjan Kristina: Institute of Psychiatric Phenomics and Genomics (IPPG), University Hospital, LMU Munich, Munich, 80336, Germany, Department of Psychiatry and Psychotherapy, University Hospital, LMU Munich, Munich, 80336, Germany; Budde Monika: Institute of Psychiatric Phenomics and Genomics (IPPG), University Hospital, LMU Munich, Munich, 80336, Germany; Comes Ashley L.: Institute of Psychiatric Phenomics and Genomics (IPPG), University Hospital, LMU Munich, Munich, 80336, Germany; Falkai; Peter: Department of Psychiatry and Psychotherapy, University Hospital, LMU Munich, Munich, 80336, Germany; Heilbronner Maria: Institute of Psychiatric Phenomics and Genomics (IPPG), University Hospital, LMU Munich, Munich, 80336, Germany; Heilbronner Urs: Institute of Psychiatric Phenomics and Genomics (IPPG), University Hospital, LMU Munich, Munich, 80336, Germany;
Kalman Janos L.: Institute of Psychiatric Phenomics and Genomics (IPPG), University Hospital, LMU Munich, Munich, 80336, Germany; Department of Psychiatry and Psychotherapy, University Hospital, LMU Munich, Munich, 80336, Germany, International Max Planck Research School for Translational Psychiatry (IMPRS-TP), Max Planck Institute of Psychiatry, Munich, 80804, Germany; Oraki Kohshour Mojtaba: Institute of Psychiatric Phenomics and Genomics (IPPG), University Hospital, LMU Munich, Munich, 80336, Germany, Department of Immunology, Faculty of Medicine, Ahvaz Jundishapur University of Medical Sciences, Ahvaz, Iran; Papiol Sergi: Institute of Psychiatric Phenomics and Genomics (IPPG), University Hospital, LMU Munich, Munich, 80336, Germany, Department of Psychiatry and Psychotherapy, University Hospital, LMU Munich, Munich, 80336, Germany; Reich-Erkelenz Daniela: Institute of Psychiatric Phenomics and Genomics (IPPG), University Hospital, LMU Munich, Munich, 80336, Germany; Schaupp Sabrina K.: Institute of Psychiatric Phenomics and Genomics (IPPG), University Hospital, LMU Munich, Munich, 80336, Germany; Schulte Eva C.: Institute of Psychiatric Phenomics and Genomics (IPPG), University Hospital, LMU Munich, Munich, 80336, Germany, Department of Psychiatry and Psychotherapy, University Hospital, LMU Munich, Munich, 80336, Germany; Schulze Thomas G.: Institute of Psychiatric Phenomics and Genomics (IPPG), University Hospital, LMU Munich, Munich, 80336, Germany; Senner Fanny: Institute of Psychiatric Phenomics and Genomics (IPPG), University Hospital, LMU Munich, Munich, 80336, Germany, Department of Psychiatry and Psychotherapy, University Hospital, LMU Munich, Munich, 80336, Germany; Vogl Thomas: Institute of Psychiatric Phenomics and Genomics (IPPG), University Hospital, LMU Munich, Munich, 80336, Germany; Anghelescu lon-George Department of Psychiatry and Psychotherapy, Mental Health Institute Berlin, Berlin, 14050, Germany; Arolt Volker: Institute for Translational Psychiatry, University of Münster, Münster, 48149, Germany; Baune Bernhardt T.: Department of Psychiatry, University of Münster, Münster, 48149, Germany: Department of Psychiatry, Melbourne Medical School, The University of Melbourne, Melbourne, Australia; The Florey Institute of Neuroscience and Mental Health, The University of Melbourne, Parkville, VIC, Australia; Dannlowski Udo: Institute for Translational Psychiatry, University of Münster, Münster, 48149, Germany; Dietrich; Detlef: AMEOS Clinical Center Hildesheim, Hildesheim, 31135, Germany, Center for Systems Neuroscience (ZSN), Hannover, 30559, Germany, Department of Psychiatry, Medical School of Hannover, Hannover, 30625, Germany; Fallgatter Andreas: Department of Psychiatry and Psychotherapy, University Tübingen, Tübingen, 72076, Germany; Figge Christian: Karl-Jaspers Clinic, European Medical School Oldenburg-Groningen, Oldenburg, 26160, Germany; Jäger Markus: Department of Psychiatry II, Ulm University, Bezirkskrankenhaus Günzburg, Günzburg, 89312, Germany; Lang Fabian: Department of Psychiatry II, Ulm University, Bezirkskrankenhaus Günzburg, Günzburg, 89312, Germany; Juckel Georg: Department of Psychiatry, Ruhr University Bochum, LWL University Hospital, Bochum, 44791, Germany; Konrad Carsten: Department of Psychiatry and Psychotherapy, Agaplesion Diakonieklinikum, Rotenburg, 27356, Germany; Reimer Jens: Department of Psychiatry and Psychotherapy, University Medical Center Hamburg-Eppendorf, Hamburg, 20246, Germany, Department of Psychiatry, Health North Hospital Group, Bremen, 28102, Germany; Reininghaus Eva: Department of Psychiatry and Psychotherapeutic Medicine, Research Unit for Bipolar Affective Disorder, Medical University of Graz, Graz, 8036, Austria; Schmauß Max: Department of Psychiatry and Psychotherapy, Bezirkskrankenhaus Augsburg, Augsburg, 86156, Germany; Schmitt Andrea:Department of Psychiatry and Psychotherapy, University Hospital, LMU Munich, Munich, 80336, Germany; Spitzer; Carsten: Department of Psychosomatic Medicine and Psychotherapy, University Medical Center Rostock, Rostock, 18147, Germany; von Hagen Martin: Clinic for Psychiatry and Psychotherapy, Clinical Center Werra-Meißner, Eschwege, 37269, Germany; Wiltfang Jens: Department of Psychiatry and Psychotherapy, University Medical Center Göttingen, Göttingen, 37075, Germany, German Center for Neurodegenerative Diseases (DZNE), Göttingen, 37075, Germany, iBiMED, 
Medical Sciences Department, University of Aveiro, Aveiro, 3810-193, Portugal; Zimmermann; Jörg: Psychiatrieverbund Oldenburger Land gGmbH, Karl-Jaspers-Klinik, Bad Zwischenahn, 26160, Germany; Andlauer Till F. M.: Department of Neurology, University Hospital rechts der Isar, School of Medicine, Technical University of Munich, Munich, 81675, Germany; Nöthen Markus M.: Institute of Human Genetics, University of Bonn School of Medicine \& University Hospital Bonn, Bonn, 53127, Germany; Degenhardt; Franziska: Institute of Human Genetics, University of Bonn School of Medicine \& University Hospital Bonn, Bonn, 53127, Germany; Forstner Andreas J.: Institute of Human Genetics, University of Bonn School of Medicine \& University Hospital Bonn, Bonn, 53127, Germany, Center for Human Genetics, University of Marburg, Marburg 35033, Germany, Department of Biomedicine, University of Basel, Basel 4031, Switzerland, Department of Psychiatry (UPK), University of Basel, Basel 4002, Switzerland; Rietschel Marcella: Department of Genetic Epidemiology in Psychiatry, Central Institute of Mental Health, Medical Faculty Mannheim, University of Heidelberg, Mannheim, 68159, Germany Germany; Witt Stephanie H.: Department of Genetic Epidemiology in Psychiatry, Central Institute of Mental Health, Medical Faculty Mannheim, University of Heidelberg, Mannheim, 68159, Germany Germany; Fischer Andre: German Center of Neurodegenerative Diseases, University of Göttingen, Göttingen, 37075, Germany.

Funding Open Access funding enabled and organized by Projekt DEAL. Please see the Supplement for full list of funding and acknowledgments.

\section{Declarations}

Conflict of interest Dr. Kato reports grants and personal fees from Japan Agency for Medical Research and Development (AMED), grants and personal fees from Ministry of Education, Culture, Sports, Science and Technology (MEXT)/Japan Society for the Promotion of Science (JSPS), personal fees from Kyowa Hakko Kirin Co., Ltd., personal fees from Eli Lilly Japan K.K., grants and personal fees from Otsuka Pharmaceutical Co., Ltd., personal fees from GlaxoSmithKline K.K., personal fees from Taisho Pharma Co., Ltd., grants and personal fees from Dainippon Sumitomo Pharma Co., Ltd., personal fees from Meiji Seika Pharma Co., Ltd., personal fees from Pfizer Japan Inc., personal fees from Mochida Pharmaceutical Co., Ltd., grants and personal fees from Shionogi \& Co., Ltd., personal fees from Janssen Pharmaceutical K.K., personal fees from Janssen Asia Pacific, personal fees from Yoshitomiyakuhin, personal fees from Astellas Pharma Inc., personal fees from Nippon Boehringer Ingelheim Co. Ltd., personal fees from MSD K.K., personal fees from Kyowa Pharmaceutical Industry Co., Ltd., grants and personal fees from Takeda Pharmaceutical Co., Ltd., personal fees from Taisho Pharmaceutical Co., Ltd., personal fees from Taisho Toyama Pharmaceutical Co., Ltd., grants and personal fees from Eisai Co., Ltd., grants and personal fees from Mitsubishi Tanabe Pharma Corporation, grants from Teijin Pharma, outside the submitted work. The other authors have no conflicts of interest to declare that are relevant to the content of this article.

Ethical approval All studies were approved by the local ethics committees.

Consent to participate All participants provided written informed consent.

Open Access This article is licensed under a Creative Commons Attribution 4.0 International License, which permits use, sharing, adaptation, distribution and reproduction in any medium or format, as long as you give appropriate credit to the original author(s) and the source, provide a link to the Creative Commons licence, and indicate if changes were made. The images or other third party material in this article are included in the article's Creative Commons licence, unless indicated otherwise in a credit line to the material. If material is not included in the article's Creative Commons licence and your intended use is not permitted by statutory regulation or exceeds the permitted use, you will need to obtain permission directly from the copyright holder. To view a copy of this licence, visit http://creativecommons.org/licenses/by/4.0/.

\section{References}

1. Zalsman G, Hawton K, Wasserman D et al (2016) Suicide prevention strategies revisited: 10-year systematic review. Lancet Psychiatry 3:646-659. https://doi.org/10.1016/S2215-0366(16) 30030-X

2. Qin P (2011) The impact of psychiatric illness on suicide: differences by diagnosis of disorders and by sex and age of subjects. $J$ Psychiatr Res 45:1445-1452. https://doi.org/10.1016/j.jpsychires. 2011.06.002

3. Mann JJ (2003) Neurobiology of suicidal behaviour. Nat Rev Neurosci 4:819-828. https://doi.org/10.1038/nrn1220

4. Voracek M, Loibl LM (2007) Genetics of suicide: a systematic review of twin studies. Wien Klin Wochenschr 119:463-475. https://doi.org/10.1007/s00508-007-0823-2

5. Brent DA, Mann JJ (2005) Family genetic studies, suicide, and suicidal behavior. Am J Med Genet Semin Med Genet 133C:13-24

6. McGuffin P, Marusic A, Farmer A (2001) What can psychiatric genetics offer suicidology? Crisis 22:61-65

7. Mullins N, Bigdeli TB, B $\emptyset$ rglum AD et al (2019) GWAS of suicide attempt in psychiatric disorders and association with major depression polygenic risk scores. Am J Psychiatry 176:651-660. https://doi.org/10.1176/appi.ajp.2019.18080957

8. Turecki G (2014) The molecular bases of the suicidal brain. Nat Rev Neurosci 15:802-816

9. Akiskal HS, Akiskal KK, Haykal RF et al (2005) TEMPS-A: progress towards validation of a self-rated clinical version of the temperament evaluation of the Memphis, Pisa, Paris, and San Diego Autoquestionnaire. J Affect Disord 85:3-16. https://doi.org/10. 1016/j.jad.2004.12.001

10. Goldberg LR (1992) The development of markers for the big-five factor structure. Psychol Assess 4:26-42. https://doi.org/10.1037/ 1040-3590.4.1.26

11. Luciano M, Hagenaars SP, Davies G et al (2018) Association analysis in over 329,000 individuals identifies 116 independent variants influencing neuroticism. Nat Genet 50:6-11. https://doi. org/10.1038/s41588-017-0013-8

12. van den Berg SM, de Moor MHM, Verweij KJH et al (2016) Metaanalysis of genome-wide association studies for extraversion: findings from the genetics of personality consortium. Behav Genet 46:170. https://doi.org/10.1007/S10519-015-9735-5

13. DeShong HL, Tucker RP, O'Keefe VM et al (2015) Five factor model traits as a predictor of suicide ideation and interpersonal suicide risk in a college sample. Psychiatry Res 226:217-223. https://doi.org/10.1016/j.psychres.2015.01.002

14. Na KS, Cho SE, Hong JP et al (2020) Association between personality traits and suicidality by age groups in a nationally representative Korean sample. Med (United States). https://doi.org/10.1097/ MD.0000000000019161

15. Blüml V, Kapusta ND, Doering S et al (2013) Personality factors and suicide risk in a representative sample of the german general population. PLoS ONE. https://doi.org/10.1371/journal.pone. 0076646 
16. Sanchez-Roige S, Gray JC, MacKillop J et al (2018) The genetics of human personality. Genes Brain Behav. https://doi.org/10.1111/ gbb.12439

17. Vukasović T, Bratko D (2015) Heritability of personality: a metaanalysis of behavior genetic studies. Psychol Bull 141:769-785. https://doi.org/10.1037/bul0000017

18. Gale CR, Hagenaars SP, Davies G et al (2016) Pleiotropy between neuroticism and physical and mental health: findings from 108038 men and women in UK biobank. Transl Psychiatry. https://doi.org/ 10.1038/tp.2016.56

19. Andlauer TFM, Nöthen MM (2020) Polygenic scores for psychiatric disease: from research tool to clinical application. Med Genet 32:39-45. https://doi.org/10.1515/medgen-2020-2006

20. Rammstedt B, John OP (2007) Measuring personality in one minute or less: a 10-item short version of the Big Five Inventory in English and German. J Res Pers 41:203-212. https://doi.org/10. 1016/j.jrp.2006.02.001

21. Costa PT Jr, McCrae RR (1992) Revised NEO Personality Inventory (NEO-PI-R) and NEO Five-Factor Inventory (NEO-FFI) professional manual. Psychol Assess Resources, Odessa, FL

22. Kalman JL, Olde Loohuis LM, Vreeker A et al (2021) Characterisation of age and polarity at onset in bipolar disorder. $\mathrm{Br} \mathrm{J}$ Psychiatry. https://doi.org/10.1192/bjp.2021.102

23. Pelin H, Ising M, Stein F et al (2021) Identification of transdiagnostic psychiatric disorder subtypes using unsupervised learning. Neuropsychopharmacology 46:1895-1905. https://doi.org/ 10.1038/s41386-021-01051-0

24. Budde M, Anderson-Schmidt H, Gade K et al (2019) A longitudinal approach to biological psychiatric research: the PsyCourse study. Am J Med Genet B Neuropsychiatr Genet 180:89-102. https://doi.org/10.1002/ajmg.b.32639

25. Ge T, Chen CY, Ni Y et al (2019) Polygenic prediction via Bayesian regression and continuous shrinkage priors. Nat Commun 10:1-10. https://doi.org/10.1038/s41467-019-09718-5

26. Erdfelder E, FAul F, Buchner A, Lang AG (2009) Statistical power analyses using $G^{*}$ Power 3.1: tests for correlation and regression analyses. Behav Res Methods 41:1149-1160. https://doi.org/10. 3758/BRM.41.4.1149

27. Palla L, Dudbridge F (2015) A fast method that uses polygenic scores to estimate the variance explained by genome-wide marker panels and the proportion of variants affecting a trait. Am J Hum Genet 97:250-259. https://doi.org/10.1016/j.ajhg.2015.06.005
28. Peters EM, John A, Bowen R et al (2018) Neuroticism and suicide in a general population cohort: results from the UK Biobank Project. BJPsych Open 4:62-68. https://doi.org/10.1192/bjo.2017.12

29. Hafferty JD, Navrady LB, Adams MJ et al (2019) The role of neuroticism in self-harm and suicidal ideation: results from two UK population-based cohorts. Soc Psychiatry Psychiatr Epidemiol 54:1505-1518. https://doi.org/10.1007/s00127-019-01725-7

30. Yen S, Shea MT, Sanislow CA et al (2009) Personality traits as prospective predictors of suicide attempts. Acta Psychiatr Scand 120:222-229. https://doi.org/10.1111/j.1600-0447.2009.01366.x

31. Sparding T, Pålsson E, Joas E et al (2017) Personality traits in bipolar disorder and influence on outcome. BMC Psychiatry. https://doi.org/10.1186/s12888-017-1332-0

32. Rappaport LM, Flint J, Kendler KS (2017) Clarifying the role of neuroticism in suicidal ideation and suicide attempt among women with major depressive disorder. Psychol Med 47:23342344. https://doi.org/10.1017/S003329171700085X

33. Su MH, Chen HC, Lu ML et al (2018) Risk profiles of personality traits for suicidality among mood disorder patients and community controls. Acta Psychiatr Scand 137:30-38. https://doi.org/10. 1111/acps.12834

34. Popovic D, Benabarre A, Crespo JM et al (2014) Risk factors for suicide in schizophrenia: systematic review and clinical recommendations. Acta Psychiatr Scand 130:418-426. https://doi.org/ 10.1111/acps. 12332

35. Brezo J, Paris J, Turecki G (2006) Personality traits as correlates of suicidal ideation, suicide attempts, and suicide completions: a systematic review. Acta Psychiatr Scand 113:180-206

36. Russell AE, Hemani G, Jones HJ et al (2021) An exploration of the genetic epidemiology of non-suicidal self-harm and suicide attempt. BMC Psychiatry 21:207. https://doi.org/10.1186/ s12888-021-03216-Z

37. Bernert RA, Hom MA, Roberts LW (2014) A review of multidisciplinary clinical practice guidelines in suicide prevention: toward an emerging standard in suicide risk assessment and management, training and practice. Acad Psychiatry 38:585-592. https://doi. org/10.1007/s40596-014-0180-1

38. Kalman JL, Loohuis LMO, Vreeker A et al (2021) Characterization of age and polarity at onset in bipolar disorder. medRxiv. https://doi.org/10.1101/2021.04.16.21251163

\title{
Authors and Affiliations
}

\author{
Janos L. Kalman ${ }^{1,2,3}$ (1) $\cdot$ Tomoya Yoshida $^{4} \cdot$ Till F. M. Andlauer $^{5,6} \cdot$ Eva C. Schulte $^{1,2} \cdot$ Kristina Adorjan $^{1,2}$. \\ Martin Alda ${ }^{7} \cdot$ Raffaela Ardau $^{8}$. Jean-Michel Aubry ${ }^{9,10}$. Katharina Brosch ${ }^{11,12}$. Monika Budde ${ }^{1}$. \\ Caterina Chillotti ${ }^{8}$. Piotr M. Czerski ${ }^{13} \cdot$ Raymond J. DePaulo $^{14} \cdot$ Andreas Forstner $^{15,16,17} \cdot$ Fernando S. Goes $^{14}$. \\ Maria Grigoroiu-Serbanescu ${ }^{18}$. Paul Grof ${ }^{19,20}$. Dominik Grotegerd ${ }^{21}$. Tim Hahn ${ }^{21}$. Maria Heilbronner ${ }^{1}$. \\ Roland Hasler ${ }^{9}$. Urs Heilbronner ${ }^{1}$. Stefanie Heilmann-Heimbach ${ }^{16}$. Pawel Kapelski ${ }^{13}$. Tadafumi Kato ${ }^{22}$. \\ Mojtaba Oraki Kohshour ${ }^{1,23}$. Susanne Meinert ${ }^{21,24} \cdot$ Tina Meller $^{11,12} \cdot$ Igor Nenadić $^{11,12} \cdot$ Markus M. Nöthen $^{16}$. \\ Tomas Novak ${ }^{25,26}$. Nils Opel ${ }^{21}$. Joanna Pawlak ${ }^{13}$. Julia-Katharina Pfarr ${ }^{11,12}$. James B. Potash ${ }^{14}$. \\ Daniela Reich-Erkelenz ${ }^{1}$. Jonathan Repple ${ }^{21} \cdot$ Hélène Richard-Lepouriel $I^{9} \cdot$ Marcella Rietschel $^{27}$. \\ Kai G. Ringwald ${ }^{11,12}$. Guy Rouleau ${ }^{28}$. Sabrina Schaupp ${ }^{1} \cdot$ Fanny Senner $^{1,2} \cdot$ Giovanni Severino $^{29}$. \\ Alessio Squassina ${ }^{29} \cdot$ Frederike Stein $^{11,12} \cdot$ Pavla Stopkova $^{25,26} \cdot$ Fabian Streit $^{27} \cdot$ Katharina Thiel $^{21}$. \\ Florian Thomas-Odenthal ${ }^{11}$. Gustavo Turecki ${ }^{30}$. Joanna Twarowska-Hauser ${ }^{13} \cdot$ Alexandra Winter $^{21} \cdot$ Peter P. Zandi $^{14}$. \\ John R. Kelsoe ${ }^{31}$. Consortium on Lithium Genetics (ConLiGen), PsyCourse · Peter Falkai ${ }^{2} \cdot$ Udo Dannlowski ${ }^{21}$. \\ Tilo Kircher ${ }^{11,12} \cdot$ Thomas G. Schulze ${ }^{1,14,32} \cdot$ Sergi Papiol ${ }^{1,2,33}$
}


1 Institute of Psychiatric Phenomics and Genomics (IPPG), University Hospital, LMU Munich, Nussbaumstr. 7, 80336 Munich, Germany

2 Department of Psychiatry and Psychotherapy, University Hospital Munich, Munich, Germany

3 International Max Planck Research School for Translational Psychiatry, Munich, Germany

4 National Center for Global Health and Medicine, Tokyo, Japan

5 Department of Neurology, Klinikum Rechts Der Isar, School of Medicine, Technical University of Munich, Munich, Germany

6 Global Computational Biology and Data Sciences, Boehringer Ingelheim Pharma GmbH \& Co. KG, 88397 Biberach an der Riß, Germany

7 Department of Psychiatry, Dalhousie University, Halifax, Canada

8 Unit of Clinical Pharmacology, University Hospital Agency of Cagliari, Cagliari, Italy

9 Department of Psychiatry, Geneva University Hospitals, Geneva, Switzerland

10 Faculty of Medicine, University of Geneva, Geneva, Switzerland

11 Department of Psychiatry and Psychotherapy, University of Marburg, Marburg, Germany

12 Center for Mind, Brain and Behavior, University of Marburg, Marburg, Germany

13 Department of Psychiatric Genetics, Poznan University of Medical Sciences, Poznan, Poland

14 Department of Psychiatry and Behavioral Sciences, Johns Hopkins University School of Medicine, Baltimore, MD, USA

15 Centre for Human Genetics, University of Marburg, Marburg, Germany

16

Institute of Human Genetics, School of Medicine \&, University of Bonn, University Hospital Bonn, Bonn, Germany
17 Institute of Neuroscience and Medicine (INM-1), Research Centre Jülich, Jülich, Germany

18 Psychiatric Genetics Research Unit, Alexandru Obregia Clinical Psychiatric Hospital, Bucharest, Romania

19 Mood Disorders Clinic of Ottawa, Ottawa, ON, Canada

20 Department of Psychiatry, University of Toronto, Toronto, ON, Canada

21 Institute for Translational Psychiatry, University of Munster, Munster, Germany

22 Department of Psychiatry and Behavioral Science, Juntendo University Graduate School of Medicine, Tokyo, Japan

23 Department of Immunology, School of Medicine, Ahvaz Jundishapur University of Medical Sciences, Ahvaz, Iran

24 Institute for Translational Neuroscience, University of Münster, Munster, Germany

25 National Institute of Mental Health, Klecany, Czech Republic

26 3Rd Faculty of Medicine, Charles University, Prague, Czech Republic

27 Department of Genetic Epidemiology in Psychiatry, Medical Faculty Mannheim, Central Institute of Mental Health, Heidelberg University, Mannheim, Germany

28 Montreal Neurological Institute, McGill University, Montreal, Canada

29 Department of Biomedical Sciences, University of Cagliari, Cagliari, Italy

30 The Douglas Research Centre, McGill University, Montreal, Canada

31 Department of Psychiatry, University of California San Diego, La Jolla, CA, USA

32 Department of Psychiatry and Behavioral Sciences, SUNY Upstate Medical University, Syracuse, NY, USA

33 Centro de Investigación Biomedica en Red de Salud Mental (CIBERSAM), Barcelona, Spain 